\title{
APORTES DE ANA MARÍA LORANDI. UNA MIRADA DESDE LA FORMACIÓN DE GRADO EN ANTROPOLOGÍA: LA CÁTEDRA FUNDADA Y SU DEVENIR
}

\author{
CONTRIBUTIONS BY ANA MARÍA LORANDI. A LOOK FROM \\ THE FORMATION OF DEGREE IN ANTHROPOLOGY: \\ THE FOUNDING CHAIR AND ITS BECOMING
}

\author{
Roxana García Sanz
}

\begin{abstract}
El presente artículo propone una recuperación de los aportes de Ana María Lorandi a la formación de grado en Antropología social, desde la perspectiva de una exestudiante de la materia Sistemas Socioculturales de América II que fuera fundada por ella en 1984. El enfoque está puesto en la revisión del inicio de la cátedra, en las novedades que introduce para la formación en Antropología y en su devenir hasta la actualidad. Asimismo, se destacan la relación con los estudiantes y su incidencia en la formación de investigadores a partir de la misma.
\end{abstract}

Palabras claves: Ana María Lorandi, formación de grado en Antropología, cátedras, Universidad de Buenos Aires.

The present article proposes a recovery of the contributions of Ana María Lorandi to the formation of degree in social anthropology, from the perspective of student of the current subject that was founded by her in 1984. The focus is then on the review of the beginning ff the Chair and the novelties that it introduces for the formation in anthropology and in its evolution until the present time. In addition, the relationship with the students and its incidence in the training of researchers from the same stand out.

Key words: Ana María Lorandi, Anthropology degree training, chairs, Universidad de Buenos Aires.

\section{Introducción}

Nos proponemos recuperar en este trabajo algunos de los aportes de Ana María Lorandi a la formación de grado en antropología, a partir de la revisión de la cátedra Etnografía Americana y Argentina fundada por ella en 1984 y su continuación como Sistemas Socioculturales de América II en la actualidad. Dicha materia, que corresponde a la orientación sociocultural de la formación de grado en Antropología introduce, por primera vez en la carrera, a la Etnohistoria como área plausible de desarrollo a partir de una relación dialéctica entre la Antropología y la Historia. Esto presenta una propuesta novedosa, tanto por su metodología (el trabajo con fuentes históricas) como por la perspectiva de análisis americanista en el área andina (la que incluye el Tucumán colonial como nuevo espacio de investigación) y Mesoamérica.

Por su parte, el abordaje de los contenidos de la materia, a partir de pensar a los actores sociales y sus relaciones en un entramado de poder en un contexto histórico determinado, nos permite además de la comprensión fáctica, desde el período precolonial hasta la conformación de los Estados nacionales, una mirada que sitúa a los grupos sociales subalternizados como actores políticos que implementan creativamente estrategias de acción/ resistencia frente a los cambios producidos en estas sociedades preestatales y estatales, frente al avance del Estado español.

\section{Los antecedentes de la cátedra}

Puede pensarse como antecedente inmediato de la cátedra la materia acerca de etnografía de Tierras Altas, dictada por Jehan Vellard, desde fines de los 60 hasta 1983. A su vez, los antecedentes del médico, etnógrafo y naturalista Vellard en el campo de los estudios andinos deben buscarse en relación con otros dos investigadores franceses: el geógrafo Marc Pieyre (Universidad de San Marcos, Perú) y el arqueólogo Henri Reichleri (director del Museo del Hombre, París), quienes se reunieron para dar forma a un centro de estudios andinos hacia la década de 1940. Robert Bazin, en la embajada francesa

\footnotetext{
* Universidad de Buenos Aires, Facultad de Filosofía y Letras. Instituto de Ciencias Antropológicas. Sección Antropología Social. Buenos Aires, Argentina. Correo electrónico: roxanagsanz@gmail.com
} 
en Perú, apoyó la iniciativa a raíz de su interés en reforzar el prestigio de Francia en la región, frente al creciente monopolio científico norteamericano tras la Segunda Guerra Mundial. Finalmente, el Centro Francés de Estudios Andinos se creó en 1948, bajo la dirección de Vellard, constituido por tres secciones: Ciencias Biológicas, Ciencias Geográficas y Ciencias Antropológicas. Los objetivos incluían las investigaciones pluridisciplinarias en terreno, el intercambio entre investigadores de las academias de los países andinos y de Francia y la formación de jóvenes investigadores locales. Vellard, quien fue director del citado Centro por 14 años, fue además una figura destacada tanto en la investigación como en la dirección de instituciones -muchas veces francesas- en distintos países de América del Sur. Su primer destino fue Brasil, donde a fines de la década de 1920 se incorporó al Instituto Butantan, acompañando en 1935 a Claude Lévi-Strauss en su expedición al Mato Grosso. En 1940 fue nombrado director del Museo Nacional de la Paz, en 1944 fue designado Jefe del Departamento de Zoología de la Universidad de Tucumán y en 1947 obtuvo el cargo de director del Museo de Historia Natural de La Paz. Desde 1953 y hasta inicios de la década siguiente dirigió el Seminario de Antropología que se estableció en el Instituto Riva Agüero de la Pontificia Universidad Católica del Perú (PUCP). En 1962 Vellard dejó el Centro Francés de Estudios Andinos porque fue enviado a dirigir el Instituto Boliviano de Biología de Altura, recientemente creado en La Paz. Luego, en 1968, se incorporó como investigador al Instituto de Ciencias Antropológicas de la Facultad de Filosofía y Letras de la Universidad de Buenos Aires y dirigió desde allí un programa de investigación etnográfica para el Gran Chaco (Ramos 2016: 36-37).

\section{El programa inicial de la cátedra Lorandi (1984)}

Ana María Lorandi, graduada en Historia en la Universidad del Litoral en 1960, se inició tempranamente, desde su formación de grado, en la investigación arqueológica. Ganó el concurso como docente auxiliar y participó de la organización del primer Instituto de Antropología de Rosario. A fines de la década del 60, ya doctorada, ingresó a la carrera de investigadora de CONICET. Luego de la renuncia colectiva de docentes en algunas universidades nacionales como consecuencia de los sucesos de la "noche de los bastones largos", se trasladó a Buenos Aires y se incorporó como investigadora al Museo de Ciencias Naturales de la Universidad Nacional de la Plata, con el arqueólogo Rex González. En esa Universidad obtuvo el cargo de Profesora en la materia Arqueología Americana II, docencia que ejerció hasta fines de 1983, pasando algunos períodos en Francia, donde se abocó a su formación etnohistórica. En 1984 fue convocada por Carlos Herrán, director del Departamento de Ciencias Antropológicas de la Universidad de Buenos Aires para dirigir el Instituto de Ciencias Antropológicas (ICA), en el contexto de restablecimiento de la democracia en el que se inicia un proceso de reestructuración de la carrera en la Facultad de Filosofía y Letras. Bajo la dirección de Lorandi, el ICA quedó conformado por cinco secciones: Etnología y Folklore, Antropología Biológica, Arqueología, Antropología Social y Etnohistoria. El equipo de esta última, dirigido por Lorandi, es el fundador de la cátedra Etnografía Americana y Argentina que introduce como novedad en la carrera el estudio de las sociedades indígenas de Mesoamérica y Andes. Junto con Ana María Presta y Mercedes del Río, Lorandi elaboró el programa de la materia que incluía entre sus temas de estudio: aspectos teóricos básicos como concepto de Etnohistoria, enfoque multidisciplinario, técnicas auxiliares; la Ecología: pisos ecológicos, diferencias latitudinales, relación con la dinámica ocupacional, etnocategorías espaciales; las Estructuras sociopolíticas básicas y sus mecanismos de interacción; los Modelos de interacción económica; el Desarrollo del Estado Incaico; las relaciones entre el Estado y las comunidades; La Religión y las cosmogonías andinas; el periodo Hispano Indígena; la Sociedad campesina, la Estructura socioeconómica de Mesoamérica: enfoque comparativo con el mundo andino. Dentro de la bibliografía de la materia se destacaban autores como: Godellier, Mauss, Espinoza Soriano, Murra, Rowe, Zuidema, Wachtel, Salomon, Pease, Saignes, Julien, Carduch, Duviois y Rostorowski.

\section{El programa actual de la cátedra}

El foco de atención de la cátedra, actualmente Sistemas Socioculturales de América II, se centra en el seguimiento de las transformaciones políticas, socioeconómicas y culturales que estas sociedades atravesaron, comprendiéndolas dentro de la perspectiva de larga duración, hasta la crisis 
del orden colonial y la transición al proceso de la independencia. Durante su extensa historia, las sociedades americanas alcanzaron un desarrollo que permite conceptualizarlas como grandes Estados. Estas formaciones políticas, a su vez, entablaron diversas relaciones de coerción y cohesión con las poblaciones que fueron incorporando a su dominio. Por otra parte, la invasión española a partir de 1492 fue el inicio de sensibles alteraciones para las sociedades americanas y, al mismo tiempo, constituyó el detonador de un complejo proceso de transformación cuyas implicaciones se advierten hasta el presente. Si bien el desarrollo de la materia se focaliza sobre el área andina, el programa incluye en las primeras unidades temas relativos a la sociedad mesoamericana prehispánica y colonial temprana, con su respectiva bibliografía, que son tratados de manera general y, a la vez, comparativa. Incorpora a los autores pioneros de la disciplina junto con estudios actuales desarrollados por el equipo de la Sección Etnohistoria.

Entre los temas que recorre se encuentran: la teoría antropológica y el contexto de surgimiento de la etnohistoria americanista; la construcción de las historias alternativas de los pueblos nativos; la organización social, económica, religiosa y política de las sociedades andinas y mesoamericanas prehispánicas; comunidades y formación de Estados; las dimensiones de la construcción de la alteridad americana en el contexto de conquista y colonización española; la formación de la sociedad andina colonial; procesos de transformación de las sociedades indígenas; el orden de la diversidad en la sociedad andina colonial; complejización sociocultural, mestizajes y las reestructuraciones, cambios y movimientos sociales en el espacio andino durante el siglo XVIII.

\section{La relación con los estudiantes de grado y posgrado}

La investigación, atrapa, la docencia, seduce, y ambas, en íntima asociación, constituyen la fuente que alimenta a la juventud

(Lorandi, 2016:21)

Respecto de la incidencia de Lorandi en la docencia, en la formación y en la investigación, tanto dentro como fuera de la Facultad de Filosofia y Letras, no podemos establecer un claro alcance, ya que sin duda ha influenciado y estimulado a varias generaciones de estudiantes. No obstante, podemos mencionar algunas de la direcciones de tesis de grado y doctorado que ha llevado a cabo, que dan cuenta de su compromiso y participación con el tiempo:

\section{Dirección de tesis de licenciatura}

Adrián, Mónica. Las doctrinas de Chayanta. Sociedad civil y clero durante la rebelión de Tomás Catari. Departamento de Ciencias Sociales, Universidad de Luján. Presentada el 30/09/1994.

Acuto, Félix. La organización del almacenaje estatal. La ocupación inka en el sector Norte del valle Calchaquí y sus alrededores. Facultad de Filosofía y Letras, UBA. Presentada en 07/11/1994.

Lamana, Gonzalo. Un proceso de construcción de identidades en el siglo XVI. Cuzco: elite nativa y elite de conquista ¿Un choque cultural? Facultad de Filosofía y Letras, UBA. Presentada el 12/12/1994.

Quarleri, Lía. Guerreros, señores y cabildantes. Conquistadores y colonizadores del Tucumán colonial 1550-1600. Facultad de Filosofía y Letras, UBA. Presentada el 30/05/1996.

Avellaneda, Mercedes. Conflicto y poder entre franciscanos y jesuitas por el control de la reducción en el Paraguay colonial. Facultad de Filosofía y Letras, UBA. Presentada el 02/10/1996.

Wilde, Guillermo. Los guaraníes después de la expulsión de los jesuitas. Procesos de construcción de diferencias socioculturales 1768-1810. Facultad de Filosofía y Letras, UBA. Presentada el 30/05/1999.

Ortemberg, Pablo. Celebraciones del poder real en Lima: simbolismo y poder en el mundo urbano colonial. Facultad de Filosofía y Letras, UBA. Presentada el 30/10/1999.

Rodríguez, Lorena. Alteraciones incaicas en el mapa étnico andino: ¿desorden controlado o caos multiétnico? El caso de los mitimaes estatales en el espacio del Chinchaysuyu. Facultad de Filosofía y Letras, UBA. Presentada el 30/06/2000.

Biondino, Claudio. Los discursos de Túpac Amaru II. Reflexiones teórico-metodológicas acerca de una historiografía conflictiva. Facultad de Filosofía y Letras, UBA. Presentada el 22/11/2002.

Justo, Silvana. Representaciones culturales en los cuentos y leyendas populares del NOA en el siglo XX. Facultad de Filosofía y Letras, UBA. Presentada el 17/03/2004.

Perusset, Macarena. Contrabando y sociedad en el Río de la Plata colonial (1580-1630). 
Facultad de Filosofía y Letras, UBA. Presentada el 22/03/2005.

Drigo, Ana Laura. Gonzalo Pizarro: liderazgo y legitimidad bajo su dirigencia en el Perú (1544-1548). Facultad de Filosofía y Letras, UBA. Presentada el 29/06/ 2005.

Cerra, Camila. Identidades en el NOA: autonomía persistente a comienzos del siglo XIX. Facultad de Filosofía y Letras, UBA. Presentada el 12/11/2009.

Cassano, Guido. Guardianes de la frontera. La población negra de Carmen de Patagones durante la primera mitad del siglo XIX. Una aproximación desde la antropología histórica. Facultad de Filosofía y Letras, UBA. Presentada el 09/09/2013

\section{Dirección de tesis de doctorado}

Williams, Verónica. Arqueología Inka de la región centro-oeste de Catamarca. Facultad de Ciencias Naturales, Universidad de La Plata. Presentada el 14/03/1996.

Nacuzzi, Lidia. Los tehuelches del norte de la Patagonia. Facultad de Filosofía y Letras, UBA. Presentada el 30/09/1996.

Del Río, Mercedes. Relaciones interétnicas y control de recursos entre los Aymaras del Macizo de Charcas. Facultad de Filosofía y Letras, UBA. Presentada el 14/11/1996

Cremonte, María Beatriz. Investigaciones arqueológicas en la Quebrada de la Ciénaga (Dpto. Tafí, Tucumán). Facultad de Ciencias Naturales, Universidad de La Plata. Presentada el 11/04/1997.

Murphy, Susana. El fenómeno de deportación asirio y neobablilónico en Samaria (720 AC.) y Jerusalem (586 AC.). Una perspectiva histórica comparada. Facultad de Filosofía y Letras, UBA. Presentada el 16/06/1999.

López de Albornoz, Cristina. La organización del espacio rural en Tucumán (1770/1820). Universidad de Tucumán. Facultad de Filosofía y Letras, Universidad Nacional de Tucumán. Presentada el 12/11/2002.

Zanolli, Carlos. Procesos de transformación de identidades/identificaciones colectivas. Jujuy. Siglos XVI y XVII. Facultad de Filosofía y Letras, UBA. Presentada el 29/08/2003.

Quarleri, Lía. Los jesuitas en Córdoba y La Rioja colonial. Construcción de poder y diferenciación y manifestaciones de oposición y resistencia en la interacción social. Facultad de Filosofía y Letras, UBA. Presentada el 05/09/2003.
Wilde, Guillermo. Antropología histórica del liderazgo guaraní misionero. 1750/1850. Facultad de Filosofía y Letras, UBA. Presentada el 15/09/2003.

Boixadós, Roxana. Parentesco e identidad en las familias de la elite riojana colonial. Facultad de Filosofía y Letras, UBA. Presentada el 12/12/2003.

Semadeni, Pablo José. La transición del estado colonial al estado republicano. Cuyo, 1770-1830. Presentada el 12/06/2008.

Avellaneda, Mercedes. Conflicto y poder: las misiones jesuíticas en las revoluciones comuneras del Paraguay. Siglos XVII y XVIII. Presentada el 10/12/2011.

El anterior listado da cuenta no solo de la incansable tarea docente de Ana María, sino también de su interés por la ampliación de las temáticas, áreas de estudio y abordajes teórico-metodológicos. En efecto, por un lado, en el desarrollo de la Sección Etnohistoria se evidencia una incorporación constante de nuevas áreas de investigación que van más allá de lo andino, como la de Pampa-Patagonia, el Noreste argentino, Río de la Plata y Paraguay. Y -por el otro- una búsqueda teórico-metodológica, proceso en el que se inscribe el reemplazo del concepto etnohistoria por el de Antropología histórica. A este respecto, señala Alejandra Ramos que la especificidad de la misma radica en atender simultáneamente a las lógicas nativas y a la profundidad temporal para abordar el estudio y la compresión de los procesos sociales. Se parte de la idea que existen diversas formas colectivas de interpretar y accionar en el mundo que pueden ser desentrañadas a partir de una participación prolongada en la vida cotidiana de los sujetos; y que estas lógicas no son inmutables, sino que se transforman a lo largo del tiempo en un interjuego entre capacidad de acción y condicionamientos que la limitan. En definitiva, la propuesta desde esta línea de la Antropología es que para comprender cabalmente las problemáticas de nuestro tiempo es imprescindible analizarlas atendiendo a la profundidad de su conformación histórica y poniendo en tensión nuestras propias categorías de análisis con aquellas que surgen de la investigación (Ramos 2016).

\section{El legado de Lorandi}

Los equipos de cátedra, las investigaciones, la conformación de equipos de investigación, la Sección Etnohistoria y sus relaciones con otros equipos locales/externos, los congresos y 
jornadas, los seminarios temáticos, son tan solo algunos títulos plausibles de ser llenados en virtud de conocer y reconocer a una pionera como Ana María Lorandi. No obstante, en estas breves líneas hemos querido recuperar una mirada que reponga la importancia de sus aportes en la formación de grado en Antropología, a lo largo de los 33 años de existencia de la materia Sistemas Socioculturales de América II, desde la perspectiva de quien fuera su alumna, incorporando de alguna manera aquellas reflexiones acerca de la materia compartidas con otros estudiantes que transitamos la cursada hacia el final de la carrera. En mi caso particular, sin dedicarme a la Antropología histórica, sino a la Antropología política y jurídica e interesada por las formas de resolución de conflictos, encuentro en mis tareas cotidianas aquella impronta particular de Ana acerca de las transformaciones políticas, económicas y culturales de los grupos inmersos en relaciones sociales asimétricas como sus señalamientos metodológicos a la hora del trabajo con fuentes. Y es justamente su "huella" la que me ha llevado a participar en este trabajo. A riesgo de ser reiterativa, como sostiene Zanolli (2016: 16), "claramente hay un antes y un después de Ana María Lorandi en la actividad académica e institucional argentina relacionada con la antropología y la etnohistoria", y es un ejercicio reflexivo y político señalar su trayectoria y los aportes que nos ha dejado desde lugares disímiles.

\section{Agradecimientos}

Agradezco a Ana María Lorandi por la motivación y la transmisión de una forma de hacer antropología con compromiso y pasión; a Carlos Chiappe por las consideraciones realizadas respecto de este texto que me permitieron mejorar el mismo; y a Jorgelina Passo por la revisión formal del mismo.

\section{Referencias Citadas}

Chiappe, Carlos María

2016 "Imaginarios en pugna: la politización del campo de los Estudios Andinos en el período fundacional de la Etnohistoria Andina”. Revista Diálogo Andino No 49.

Costilla, Julia

2016 "Itinerarios antropológicos para una etnohistoria comparada de la religión: cultos católicos americanos en la larga duración". Revista Diálogo Andino No 49.

Lorandi, A

2016 "Reflexiones acerca de una experiencia vital". En Disciplinas sin fronteras. Homenaje a Ana María Lorandi, editado por R. Boixadós y C. Busnter, pp. 21-30. EFFyL, Buenos Aires.

Ramos, A.

2016 El desarrollo de la Etnohistoria andina como campo interdisciplinar: interacciones entre Historia, Arqueología y Antropología, (Perú, Bolivia y Argentina, 1970-2005). Tesis doctoral. Facultad de Filosofía y Letras, Universidad de Buenos Aires, Buenos Aires.

Zanolli, C.

2016 "Introducción”. En Disciplinas sin fronteras. Homenaje a Ana María Lorandi, editado por R. Boixadós y C. Busnter, pp. 15-19. EFFyL, Buenos Aires.
Zanolli, C.; A. Ramos y C. Chiappe. C.

2016 "Itinerarios académicos de Ana María Lorandi". En Disciplinas sin fronteras. Homenaje a Ana María Lorandi, editado por R. Boixadós y C. Busnter, pp. 183-216. EFFyL, Buenos Aires.

Zanolli, Carlos; Ramos, Alejandra; Estruch, Dolores y Costilla, Julia

2016 "La Etnohistoria en la Universidad de Buenos Aires: un recorrido por 30 años de investigaciones”. Revista Diálogo Andino $\mathrm{N}^{\circ} 49$.

\section{Documentos citados}

Programa de la Cátedra Etnografía Americana y Argentina 1984. Facultad de Filosofía y Letras. Universidad de Buenos Aires. Archivo Institucional Sección Etnohistoria.

Programa de la Cátedra Sistemas Socioculturales de América II. 2016. Facultad de Filosofía y Letras. Universidad de Buenos Aires. Archivo Institucional Sección Etnohistoria. 
